\title{
Visualisation Tool for Complex 3D Fractals on Volumetric Displays
}

\author{
Ryosuke Arai \\ Tokyo Denki University \\ 5, Senjuasahicho, Adachi-ku, Tokyo, \\ Japan \\ 19kmh02@ms.dendai.ac.jp
}

\author{
Ou Yamamoto \\ Tokyo Denki University \\ 5, Senjuasahicho, Adachi-ku, Tokyo, \\ Japan \\ ou@mail.dendai.ac.jp
}

\begin{abstract}
Observing complex 3D fractals, such as Mandelbulb and Mandexbox, and examining their interesting details is very useful in using them for visual effects in media works, 3D arts, etc. However, the observation on a 2D display is different from their actual three-dimensional appearance. In this paper, we used a 3D volumetric display to visualise them in real 3D space. Furthermore, these 3D fractals were visualised on a commercially available stereoscopic display, and the differences in stereoscopic effect were compared.
\end{abstract}

2D and 3D Imaging. Display and Printing.

\section{INTRODUCTION}

3D fractals, such as Mandelbulb and Mandelbox, have attracted attention in recent years due to their complex and interesting details, and are useful for visual expression in media works. The original Mandelbrot set is a 2D fractal with complex convergence properties, represented by a very simple recurrence formula.

$$
z \mapsto z^{2}+c
$$

The set of $c$ that does not diverge to infinity is the Mandelbrot set. As shown in Figure 1, the set has a fractal shape on the complex plane.

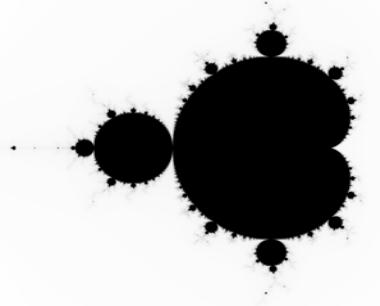

Figure 1: Mandelbrot set rendered by WebGL.

Daniel White and Paul Nylander constructed the Mandelbulb. Their idea is that instead of rotating around a circle for complex multiplication, as in a normal 2D Mandelbrot set, rotation may occur around 3D spherical coordinates (White 2009).
The formula is as follows:

$$
\begin{aligned}
& z \mapsto z^{\mathrm{n}}+c, z \in \mathbb{R}^{3} \\
& z^{n}=[x, y, z]^{n}=r^{n}[\sin n \theta \cos n \varphi, \sin n \theta \sin n \varphi, \cos n \theta]
\end{aligned}
$$

where $r, \theta, \varphi$ are the spherical coordinates of $x, y$, $z$. $n=8$ provides a typical Mandelbulb.

The Mandelbox discovered by Tom Lowe (Lowe 2010) is a box-like fractal. The formula showing the Mandelbox is as follows:

$$
v \mapsto s \operatorname{ballFold}(r, f \text { boxFold }(v))+\mathrm{c}
$$

The following Algorithm 1 and Algorithm 2 define the boxFold and ballFold procedures, respectively.

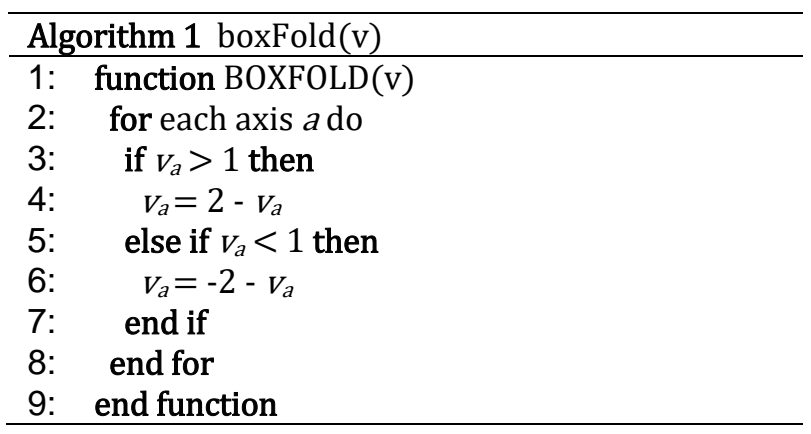




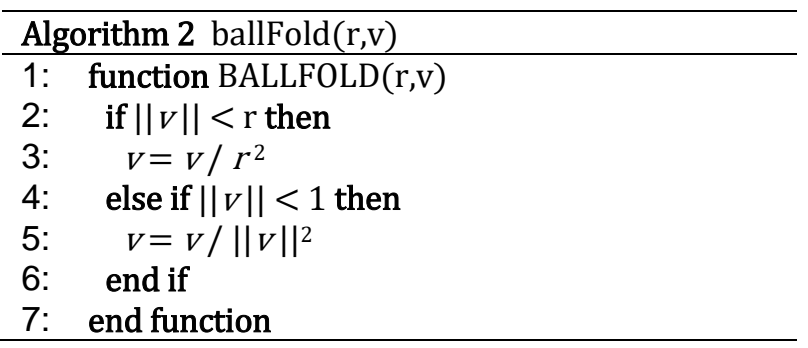

Where, $r=0.5, s=2, f=1$ provides a typical Mandelbox.

Figure 2 shows a typical Mandelbulb and Mandelbox.

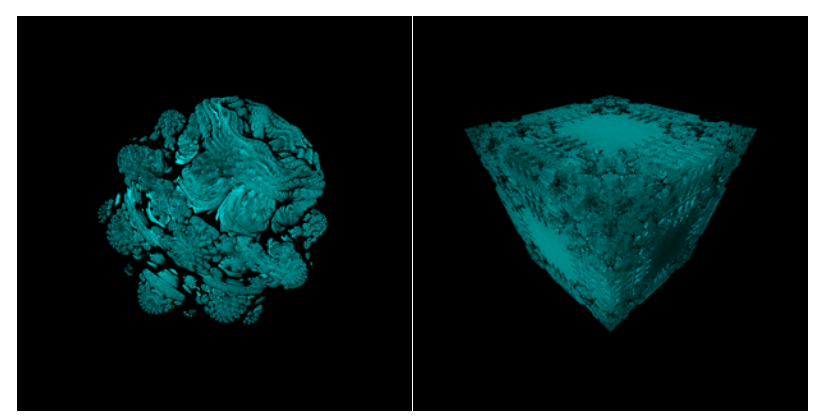

Figure 2: Mandelbulb (left) and Mandelbox (right).

As shown in Figure 2, they have complex shapes with a three-dimensional and infinite recursive structure. In addition, they have a property of performing complex transformations by changing parameters. Such visualisations are rendered using ray marching with distance functions, and recent PC performance improvements have made it possible to run in a practical amount of time, and many software tools for visualisation, such as Mandelbulber (Mandelbulber.com) have been developed.

However, in most cases, those fractals are visualised on a 2D PC display or commercial parallax type 3D display, and there are few examples of 3D visualisation that satisfy physiological factors of stereoscopic vision. Therefore, in this article, we have developed a software tool to visualise these 3D fractals so as to satisfy the physiological factors of stereoscopic vision using a volume scanning type display developed as part of our previous work (Yamamoto 2009; Yamamoto \& Kokubu 2010). We also compared the stereoscopic effect of our volumetric display with the commercial parallax type 3D display.

\section{OUR VOLUMETRIC DISPLAY}

A volumetric display is a device that creates $3 D$ images in real 3D space (Blundell \& Schwarz 2000). Most volumetric displays satisfy the physiological factors for stereoscopic viewing while requiring no $3 \mathrm{D}$ goggles or head-mounted displays. To date, several types of volumetric displays have been proposed and developed, including a variable-focal type (Suyama et al. 2000), a movingscreen type (Favalora et al. 2002, Sullivan 2004, VoxonVX1), an up-conversion type (Dowing et al. 1996, Refai 2009), and a plasma-emission type (Saito et al. 2008). Some of these are commercially available; however, they are expensive for researchers who are developing applications for them.

Our volumetric display creates sequential crosssectional images of 3D objects on Vacuum Fluorescent Display (VFD) moving in reciprocating motion; the 3D images of the objects appear because of the afterimage effect. Figure 3 shows a schematic overview of our display, the reciprocating mechanism, and an example 3D image on our display.
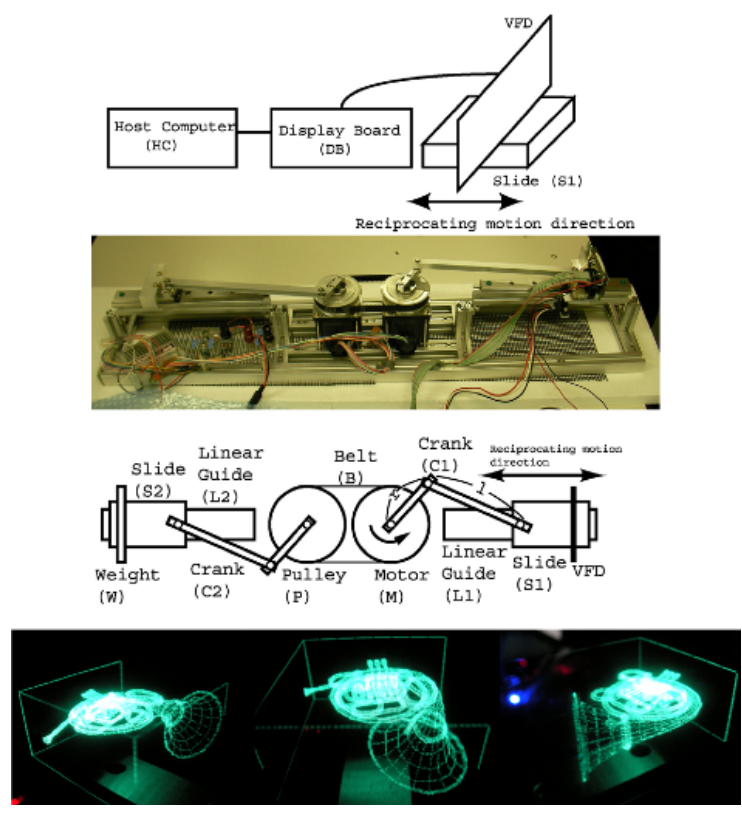

Figure 3: (Above to below) Schematic overview of our display, schematic and photo of the reciprocating mechanism. Sample 3D images on the display from three different viewpoints.

In Figure 3 , the rotation of the servomotor $M$ is transmitted as a reciprocating motion to the slide $\mathrm{S} 1$ of the linear guide L1 via the crank $\mathrm{C} 1$. Since the reciprocating motion is performed at $12 \mathrm{~Hz}$, the rotational speed of $M$ is constant at $12 \mathrm{rpms}$ (720 $\mathrm{rpm})$. The dimension of the crank: $r=3 \mathrm{~cm}$ and $\mathrm{I}=$ $20 \mathrm{~cm}$, makes the stroke of the reciprocating motion $6 \mathrm{~cm}$. VFD is fixed to S1 so that the display surface is perpendicular to figure plane. The rotation of $M$ is also transmitted to the pulley $P$ via the belt $B$ and is further transmitted to the slide S2 via the crank C2. The motion of $S 2$ is in the opposite direction to $\mathrm{S} 1$ and cancels the vibration due to $\mathrm{S} 1 . \mathrm{W}$ is an aluminium block of the same mass (about $60 \mathrm{~g}$ ) as VFD. 


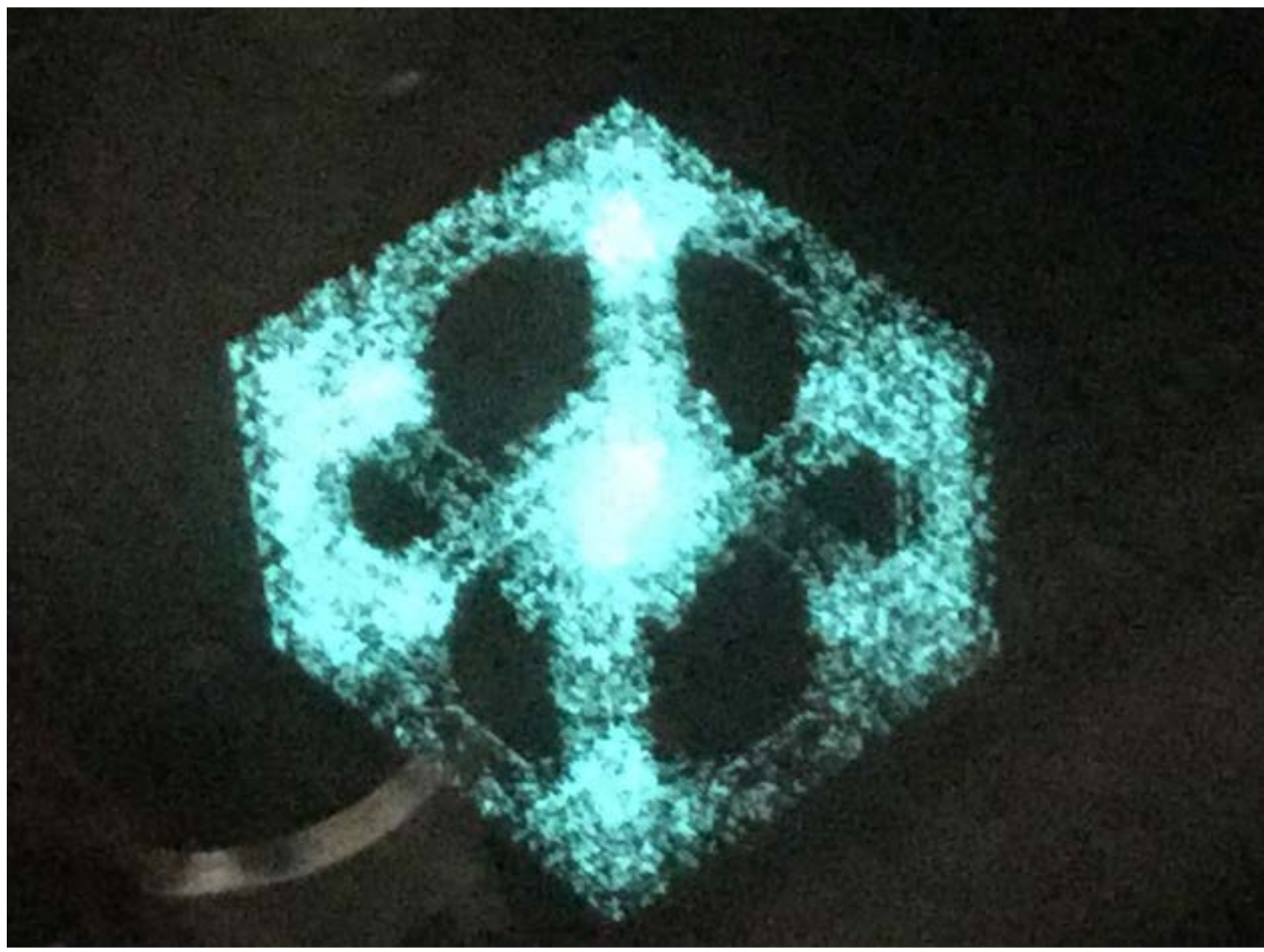

Figure 4: Mandelbox on our volumetric display

Volumetric scan is performed at the centre $4 \mathrm{~cm}$ of $6 \mathrm{~cm}$ stroke of reciprocating motion. Since the reciprocating frequency is $12 \mathrm{~Hz}$ and the scanning is performed in both forward and return, the refresh rate is 24 volumes per second which enables smooth animation.

The VFD is a commercially available, W128 $\times \mathrm{H} 64$ resolution, $0.31 \mathrm{~mm}$ pixel pitch, static drive type (Noritake). Volumetric scanning is done in the direction perpendicular to the VFD surface and each volume consists of 128 cross-sectional images displayed on VFD. Therefore, 3D images displayed with a resolution of W128 $\times \mathrm{H} 64 \times \mathrm{D} 128$ voxels in the space of $\mathrm{W} 4 \mathrm{~cm} \times \mathrm{H} 2 \mathrm{~cm} \times \mathrm{D} 4 \mathrm{~cm}$.

Display data is stored in the host computer $\mathrm{HC}$ and transferred to the display board DB via USB $2.0 \mathrm{l} / \mathrm{F}$. DB temporarily stores the data in its memory and sends the data to the VFD in synchronisation with the volumetric scan.

The display has a small display area of $(\mathrm{W} \times \mathrm{H} \times \mathrm{D})$ $4 \mathrm{~cm} \times 2 \mathrm{~cm} \times 4 \mathrm{~cm}$. However, despite the small display space, our display has sufficient resolution to display a single 3D object, about 170-degree field of view. Since the scanning is performed directly using the VFD surface without any screens or lenses, $0.3 \mathrm{~mm}$ size voxels are displayed clearly without crosstalk and geometric distortion accompanying viewpoint movement. Therefore, resultant $3 \mathrm{D}$ images are delicate and provide a strong feeling of presence at the same time. This is a unique feature of our display.

\section{OUR APPLICATION TOOL}

Our application tool was implemented using Qt Creator 4.8 and Qt 5.1.2 programming. It provides a preview area for resultant 3D fractals and parameter setting areas for the fractals, preview, and animation. A user creates a fractal while changing various parameters and setting parameters for animation. Then, the application generates animation data based on the parameters.

On the preview area, the user can freely observe fractals by mouse dragging. Once a calculation range is specified, calculations outside the range 
will be skipped. Therefore, the final execution time can be reduced.

The range specified in the render range area is automatically adjusted to the actual display area of our volumetric display. The start button and the end button set the parameter values at the start and the end point of an animation.

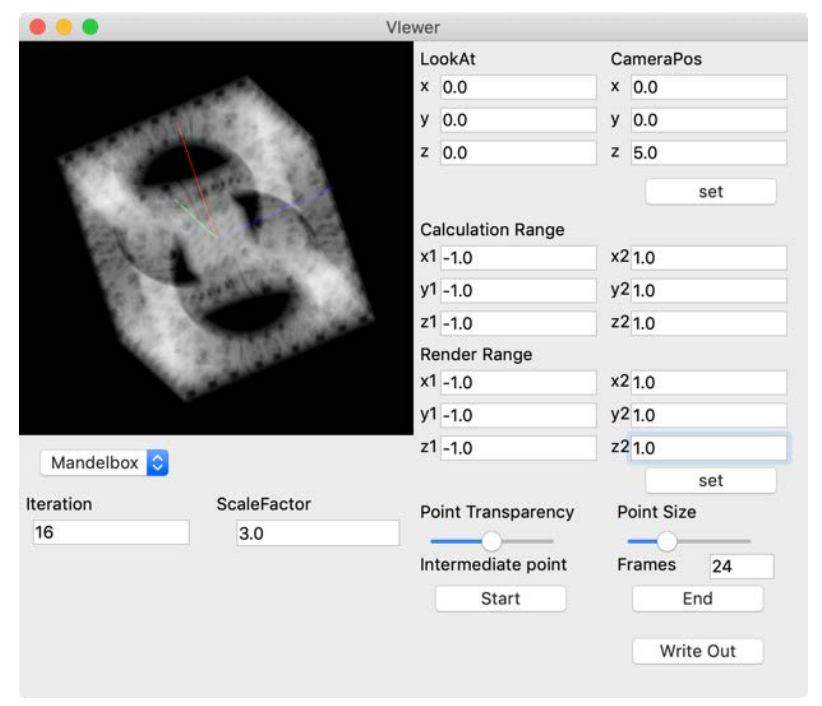

Figure 5: Our application appearance.

\section{EVALUTION AND DISCUSSION}

Regarding 3D fractals, we evaluated the difference in ease of three-dimensionally recognising structures using a commercially available parallax type 3D display and our volumetric display. As a commercially available display, we chose Looking Glass (LookingGlassFactory). In the evaluation, we used a 3D zooming animation into the center of a Mandelbox. As the animation data for Looking Glass, a polygon model was generated based on voxel data matched to the resolution of the volumetric display $(\mathrm{W} 128 \times \mathrm{D} 128 \times \mathrm{H} 128)$. The polygon model data and an animation data were created on Unity.

Each of twenty-five participants observed 3D fractals by volumetric display, and then by Looking Glass. After observing the 3D fractals by each display, participants were asked whether our display or Looking Glass was easier to threedimensionally recognising the structure of 3D fractals. All participants are students or ordinary people without background knowledge about fractals. The participants except one answered that our display was able to observe more stereoscopically $\left(p=1.55 \times 10^{-6}\right.$, two-sided binomial test).

From this result, it can be concluded that our display is effective for three-dimensionally recognising the structure of 3D fractals. The reason for such a result is considered to be that the viewing angle of Looking Glass is narrow due to a lenticular lens, and it does not correspond to the field of view in the vertical direction and it deviates from the sense of reality. We considered that volumetric display is effective for accurate understanding of 3D structure as well as 3D fractal.

\section{CONCLUSIONS}

We have proposed and implemented a tool for visualising $3 \mathrm{D}$ fractals on a volumetric display. We further evaluated the ease in understanding structures of 3D fractals using our tool as compared to using images on a commercially available parallax type 3D display. The result shows some advantages of using our tool and display rather than using images on a parallax type 3D display.

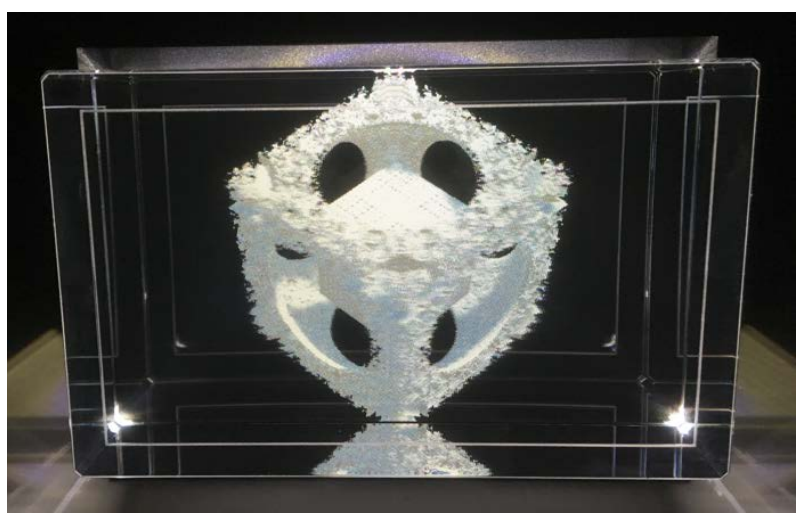

Figure 6: Mandelbox on Looking Glass.

\section{REFERENCES}

Blundell, B., Schwarz, A.: (2000) Volumetric ThreeDimensional Display Systems. Wiley-IEEE Press, New Jersey

Favalora, G., Napoli, J., Hall, D., Dorval, R., Giovinco, M., Richmond, M., Chun, W. (2002) 100 Million-voxel volumetric display. In: Proc. SPIE 4712, pp.300-312

Downing, E., Hesselink, L., Ralston, J., Macfarlane, R. (1996) A Three-Color, Solid-State, ThreeDimensional Display. Science, 273, no. 5279, pp.1185-1189

LookingGlassFactory (2020)

https://lookingglassfactory.com/ (retrieved 22 March 2020).

Lowe, T. (2010) What is a Mandelbox? https://sites.google.com/site/mandelbox/what-is-amandelbox (retrieved 20 February 2020). 
Mandelbulber.com (2020).

https://mandelbulber.com/ (retrieved 22 March 2020).

Noritake itron cl series application note apf130r1.4. https://www.noritake-

itron.jp/eng/cs/appnote/apf100 vfd/index.html (retrieved 20 February 2020).

Refai, H.H. (2009) Static Volumetric ThreeDimensional Display. J. Display Technol. 5, no. 10, pp.391-397

Saito, H., Kimura, H., Shimada, S., Naemura, T., Kayahara, J., Jarusirisawad, S., Nozick, V., Ishikawa, H., Murakami, T., Aoki, J., Asano, A., Kimura, T., Kakehata, M., Sasaki, F., Yashiro, H., Mori, M., Torizuka, K., Ino, K. (2008) Laser-plasma scanning 3D display for putting digital contents in free space. In: Proc. SPIE 6803 Stereoscopic Displays and Applications XIX, 680309

Sullivan, A. (2004) DepthCube solid state 3D volumetric display. In: Proc. SPIE 5291, pp.279284
Suyama, S., Date, M., Takata, H. (2000) ThreeDimensional Display System with Dual-Frequency Liquid-Crystal Varifocal Lens. Jpn. J. Appl. Phys. 39, pp.195-197

Skytopia (2009-2020) The Unravelling of the Real 3D Mandelbulb.

https://www.skytopia.com/project/fractal/2mandelbu lb.html (retrieved 20 February 2020).

VoxonVX1 (2020) https://voxon.co/ (retrieved 22 March 2020).

Yamamoto, O. (2009) A Volumetric Display using VFD, and A Display Data Generator (in Japanese). J. Soc. Art Sci. 8, pp.15-24

Yamamoto, O., Kokubu, M. (2010) A 3D Visualization of Isometric Deformation of Minimal Surfaces on a Volumetric Display (in Japanese). Trans. of the Visualization Society of Japan, 30, no.6, pp.39-45

White, D. (2009) The Unravelling of the Real 3D Mandelbulb. 